\title{
GENERALIZAÇÃO DE UM ÍNDICE DE INTENSIDADE DE INFECÇÃO EM EXPERIMENTOS DE AVALIAÇÃO DE DOENÇAS EM PLANTAS ${ }^{1}$
}

\begin{abstract}
ANA BEATRIZ COSTA CZERMAINSKI ${ }^{2}$
RESUMO - Em experimentos de avaliação de doenças em plantas é comum a classificação de indivíduos de cada parcela experimental, segundo classes de uma escala quantitativa discreta, mediante avaliação visual da severidade de doença. O objetivo deste trabalho foi apresentar a generalização de uma medida que associa uma resposta quantitativa denominada índice de intensidade de infecção, o qual é expresso por $\mathrm{I}=\operatorname{sen}^{2} \omega$, a cada distribuição de freqüências obtida nas parcelas, e cuja transformação angular $\omega=\operatorname{arcsen} \sqrt{\mathrm{I}}$ pode ser submetida à análise da variação. Desde que o cálculo de $\omega$ não envolve os valores ou notas da escala, ele pode ser aplicado também a uma escala qualitativa ordinal.
\end{abstract}

Termos para indexação: medida de infecção, doenças de plantas, transformação angular.

\section{GENERALIZATION OF AN INDEX OF INFECTION INTENSITY IN PLANT DISEASE EXPERIMENTS}

\begin{abstract}
In plant disease experiments, individuals of each experimental unit are commonly classified according to categories of a discrete quantitative scale through visual assessment of disease severity. This paper shows the generalization of a measure that associates a quantitative response called infection intensity index, expressed as $\mathrm{I}=\operatorname{sen}^{2} \omega$, to each distribution of frequencies obtained in the units. Its angular transformation $\omega=\operatorname{arcsen} \sqrt{\mathrm{I}}$ allows the application of the analysis of variance. Since the calculation of $\omega$ does not involve the values or notes of the scale it can also be used with a qualitative ordinal scale.
\end{abstract}

Index terms: infection measure, plant disease, angular transformation.

\section{INTRODUÇÃO}

Em experimentos fitossanitários é comum a classificação de indivíduos amostrados tais como folhas, ramos, frutos, flores, segundo notas de uma escala discreta preestabelecida, correspondentes a faixas de severidade de doença. A classificação dessas unidades amostrais em geral ocorre mediante avaliação visual e resultam na contagem de elementos ou incidência por classe. Alguns recursos estão disponíveis para a análise dessas variáveis categóricas como tabelas de contingência, onde freqüências são analisadas

\footnotetext{
${ }^{1}$ Aceito para publicação em 26 de fevereiro de 1999.

${ }^{2}$ Eng. Agr., M.Sc., Embrapa-Centro Nacional de Pesquisa de Uva e Vinho (CNPUV), Caixa Postal 130, CEP 95700-000 Bento Gonçalves, RS. E-mail: ana@cnpuv.embrapa.br
} 
ao invés de dados quantitativos (Eskridge, 1995). Porém, quando os dados provêm de um delineamento experimental, é natural a busca de um índice de intensidade de doença que represente a distribuição de freqüências obtida em cada unidade experimental, de modo a associá-la a uma resposta quantitativa que possa ser submetida à análise da variação, uni ou multivariada, ou correlacionada a outras variáveis quantitativas. O termo "intensidade", que envolve atributos de incidência e severidade, é usado aqui como uma caracterização geral de medida de doença numa área específica, conforme Seem (1984).

Em experimentos de respostas dicotômicas, isto é, com escala de valores 0 e 1, exprimindo ausência e presença de doença, a transformação angular pode ser aplicada às porcentagens de indivíduos com doença, obtidas em cada unidade experimental, para posterior análise de variação (Cochran, 1943; Bartlett, 1947). Hughes \& Madden (1995) comparam alguns métodos de avaliação de dados binários como medida de incidência.

Em escalas com três notas, ou mais, essa resposta quantitativa é obtida comumente pela média ponderada das notas, sendo também usual o cálculo de um "índice de doença”, proposto por McKinney (1923), que consiste no seguinte:

$$
\mathrm{ID}=\frac{\sum_{\mathrm{k}=1}^{\mathrm{K}} \mathrm{F}_{\mathrm{k}} \mathrm{x}_{\mathrm{k}}}{\mathrm{n} \cdot \mathrm{x}_{\mathrm{K}}}
$$

onde $\mathrm{F}_{\mathrm{k}}$ representa o número de indivíduos da amostra com nível de infecção $\mathrm{x}_{\mathrm{k}}, \mathrm{k}=1, \ldots, \mathrm{K}$, na parcela; $\mathrm{n}$ é o numero total de indivíduos na amostra; $\mathrm{x}_{\mathrm{K}}$ é o valor numérico máximo da escala empregada. $\mathrm{O}$ ID é proporcional à média ponderada das notas, pois corresponde à razão entre ela e a nota máxima da escala adotada, e pode ser expresso em porcentagem. Embora o autor tenha proposto o cálculo de ID para a escala $0,0,75,1,2$ e 3 , na avaliação de sintomas por Helminthosporium sativum em trigo, a medida é usada para outras escalas, em outros cultivos. Na literatura, encontra-se o ID submetido à análise da variação com e sem o uso da transformação angular.

Amaral (1969) alertou para a impropriedade de se submeter os dados resultantes da fórmula de McKinney (1923) à análise da variação, e propôs um índice de intensidade de infecção (I) para escalas de quatro categorias, expresso por $\mathrm{I}=\operatorname{sen}^{2} \omega$, cuja transformação angular $\omega=\operatorname{arcsen} \sqrt{\mathrm{I}}$ pode ser submetida a análise da variação e procedimentos de análise estatística subseqüentes. A variável $\omega$ é calculada por:

$\omega=0,39 \operatorname{arcsen} \sqrt{\mathrm{f}_{1}}+0,22 \sqrt{\mathrm{p}_{1,3}} \operatorname{arcsen} \sqrt{\mathrm{f}_{2}}+0,39 \sqrt{\mathrm{p}_{2,3}} \operatorname{arcsen} \sqrt{\mathrm{f}_{3}}$,

onde as freqüências relativas $f_{j}$ são frações das freqüências absolutas $\left(F_{j}\right)$, expressas por: $\mathrm{f}_{1}=\left(\mathrm{F}_{1}+\mathrm{F}_{2}+\mathrm{F}_{3}\right) / \mathrm{n}, \mathrm{f}_{2}=\left(\mathrm{F}_{2}+\mathrm{F}_{3}\right) /\left(\mathrm{F}_{1}+\mathrm{F}_{2}+\mathrm{F}_{3}\right)$ e $\mathrm{f}_{3}=\mathrm{F}_{3} /\left(\mathrm{F}_{2}+\mathrm{F}_{3}\right)$; $\mathrm{p}_{1,3}=\mathrm{p}_{1}+\mathrm{p}_{2}+\mathrm{p}_{3}$ e $\mathrm{p}_{2,3}=\mathrm{p}_{2}+\mathrm{p}_{3}$ e $\mathrm{p}_{1}, \mathrm{p}_{2}$ e $\mathrm{p}_{3}$ são as probabilidades de infecção fraca $\left(\mathrm{x}_{1}=1\right)$, regular $\left(\mathrm{x}_{2}=2\right)$ e forte $\left(\mathrm{x}_{3}=3\right)$, respectivamente, por indivíduo da parcela cujo índice de infecção está sendo determinado. Essas probabilidades são desconhecidas, e devem ser estimadas pelas freqüências relativas 
correspondentes $\mathrm{F}_{\mathrm{j}} / \mathrm{n}$. As constantes $\mathrm{k}_{1}=0,39, \mathrm{k}_{2}=0,22$ e $\mathrm{k}_{3}=0,39$ são pesos determinados a partir da atribuição do valor teórico máximo de I correspondente a cada nota da escala (Amaral, 1969). O autor propôs o índice I particularmente para escalas de quatro categorias, isto é, os pesos $\mathrm{k}_{\mathrm{j}}$ são específicos para este caso.

Silva (1969) abordou a validade estatística do índice I, constatando, através de um exemplo, a normalidade das observações e a homogeneidade de variância, requisitos estes não assegurados com o índice antigo (McKinney, 1923). Não se encontram, porém, aplicações na literatura científica, e pesquisadores seguem usando a média ponderada ou o "índice de McKinney" como medida da intensidade de doença e submetendo-os à análise da variação.

Em experimentos com videiras, por exemplo, as avaliações fitossanitárias são baseadas em escalas categóricas convencionadas distintamente para as diferentes doenças. Em caso de antracnose, utiliza-se uma escala de quatro notas $(0,1,2$ e 3$)$; para míldio nas folhas é usada uma escala de doze notas $(0,5,10(10) \ldots 100)$; para podridões no cacho (botritis, míldio e glomerela) é usada escala de seis notas (de $0=$ "sem sintoma" até $5=$ "mais de $50 \%$ de podridão"). Assim, tornou-se necessário estabelecer o cálculo de $\omega$ para escalas de mais de quatro classes.

Este trabalho teve por objetivo apresentar a generalização do índice de intensidade de infecção (I) proposto por Amaral (1969) para escalas discretas ou escalas qualitativas ordinais.

\section{MÉTODO}

A Tabela 1 representa a estrutura de respostas da classificação de $\mathrm{n}$ indivíduos por parcela, segundo $\mathrm{J}+1$ categorias $\mathrm{x}_{\mathrm{j}}, \mathrm{j}=0, \ldots, \mathrm{J}$, (uma classe de ausência de doença e $\mathrm{J}$ classes de presença de doença), onde $\mathrm{x}_{0}$ significa "ausência da doença" ou "sem sintomas" e $\mathrm{x}_{\mathrm{J}}$ a classe de intensidade máxima dentro do critério adotado. As contagens ou freqüências de indivíduos em cada classe da escala ordinal (crescente) estão representadas por $\mathrm{F}_{0}$ (número de unidades "sem infecção" ou "sem sintoma"), $\mathrm{F}_{1}$ (número de unidades no nível mais baixo de severidade de infecção), $F_{2}$, etc., até $F_{J}$ (número de unidades no nível mais alto de severidade de infecção). Por facilidade de

TABELA 1. Estrutura de respostas (freqüências absolutas) da classificação de n indivíduos por unidade experimental, segundo classes de severidade de doença $\left(x_{j}\right)$.

\begin{tabular}{lcccccc}
\hline \multirow{2}{*}{ Parcela } & \multicolumn{5}{c}{ Classe } & \multirow{2}{*}{ Total } \\
\cline { 2 - 6 } & $\mathrm{x}_{0}$ & $\mathrm{x}_{1}$ & $\mathrm{x}_{2}$ & $\ldots$ & $\mathrm{x}_{\mathrm{J}}$ & \\
\hline Parcela 1 & $\mathrm{~F}_{0}$ & $\mathrm{~F}_{1}$ & $\mathrm{~F}_{2}$ & $\ldots$ & $\mathrm{F}_{\mathrm{J}}$ & $\mathrm{n}$ \\
Parcela 2 & $\mathrm{~F}_{0}$ & $\mathrm{~F}_{1}$ & $\mathrm{~F}_{2}$ & $\ldots$ & $\mathrm{F}_{\mathrm{J}}$ & $\mathrm{n}$ \\
$\ldots$ & $\ldots$ & $\ldots$ & $\ldots$ & $\ldots$ & $\ldots$ & $\ldots$ \\
Parcela $\mathrm{p}$ & $\mathrm{F}_{0}$ & $\mathrm{~F}_{1}$ & $\mathrm{~F}_{2}$ & $\ldots$ & $\mathrm{F}_{\mathrm{J}}$ & $\mathrm{n}$ \\
\hline
\end{tabular}


demonstração algébrica e como o cálculo é efetuado por parcela, foi suprimido o índice que a representa.

Generalizando $\omega$ para escalas de $\mathrm{J}+1$ classes (uma classe referente à ausência de doença e $J$ classes de presença de doença) tem-se $\omega=\sum_{j=1}^{J} k_{j} \sqrt{p_{j-1, j}} \operatorname{arcsen} \sqrt{f_{j}}$.

Os termos $f_{j}$ e $p_{j, J}$ são obtidos dos dados observados conforme definido por Amaral (1969), ou seja, $f_{j}=\left(F_{j}+F_{j+1}+\ldots+F_{J}\right) /\left(F_{j-1}+F_{j}+F_{j+1}+\ldots+F_{J}\right), j=1, \ldots, J$ e $p_{j-1, J}=p_{j}+p_{j+1}+\ldots+p_{J}, j=1, \ldots, J$, ressaltando-se que, sendo as probabilidades $p_{j}$ estimadas por $F_{j} / n$, então $p_{0, J}=1$ e $p_{1, J}=f_{1}$. Supondo que os $n$ indivíduos de uma parcela tenham igual probabilidade de serem classificados nas $\mathrm{J}$ categorias referentes à presença de doença, o valor máximo que I assume é $\mathrm{j} / \mathrm{J}$, ou seja, para a classe $\mathrm{x}_{1}, \max (\mathrm{I})=1 / \mathrm{J} ; \operatorname{para} \mathrm{x}_{2}, \max (\mathrm{I})=2 / \mathrm{J} ; \ldots ; \operatorname{para} \mathrm{x}_{\mathrm{J}}, \max (\mathrm{I})=1$. Note-se que, se os $\mathrm{n}$ indivíduos forem classificados em $\mathrm{x}_{0}, \mathrm{I}=0$ e $\omega=0$. Estabelecidos os valores máximos $\mathrm{I}=\mathrm{j} / \mathrm{J}$, determinam-se os valores de $\omega=\operatorname{arcsen} \sqrt{\mathrm{j} / \mathrm{J}}$. Mas, como $\omega=\sum_{\mathrm{j}=1}^{\mathrm{J}} \mathrm{k}_{\mathrm{j}} \sqrt{\mathrm{p}_{\mathrm{j}-1, \mathrm{~J}}} \operatorname{arcsen} \sqrt{\mathrm{f}_{\mathrm{j}}}$, resulta que $\operatorname{arcsen} \sqrt{\mathrm{j} / \mathrm{J}}-\sum_{\mathrm{j}=1}^{\mathrm{J}} \mathrm{k}_{\mathrm{j}} \sqrt{\mathrm{p}_{\mathrm{j}-1, \mathrm{~J}}} \operatorname{arcsen} \sqrt{\mathrm{f}_{\mathrm{j}}}=0$.

Na hipótese de $\max (\mathrm{I})$, para $\mathrm{j}$ fixado $\left(\mathrm{j}^{\prime}\right), \mathrm{p}_{\mathrm{j}^{\prime}-1, \mathrm{~J}}=1$ e $\mathrm{f}_{\mathrm{j}}=1$ e a igualdade anterior fica $\operatorname{arcsen} \sqrt{\mathrm{j}^{\prime} / \mathrm{J}}-90 \sum_{\mathrm{j} \leq \mathrm{j}^{\prime}} \mathrm{k}_{\mathrm{j}}=0$. Ou, o que é o mesmo,

$\mathrm{k}_{1}=(\operatorname{arcsen} \sqrt{1 / \mathrm{J}}) / 90$

$\mathrm{k}_{2}=(\operatorname{arcsen} \sqrt{2 / \mathrm{J}}) / 90-\mathrm{k}_{1}$

$\mathrm{k}_{3}=(\operatorname{arcsen} \sqrt{3 / \mathrm{J}}) / 90-\mathrm{k}_{1}-\mathrm{k}_{2}$

$\mathrm{k}_{\mathrm{J}}=(\operatorname{arcsen} \sqrt{\mathrm{J} / \mathrm{J}}) / 90-\mathrm{k}_{1}-\mathrm{k}_{2}-\ldots-\mathrm{k}_{\mathrm{J}-1}=1-\sum_{\mathrm{j}=1}^{\mathrm{J}-1} \mathrm{k}_{\mathrm{j}}$.

A partir desse sistema de $\mathbf{J}$ equações e $\mathrm{J}$ incógnitas, são calculados, seqüencialmente, os valores $\mathrm{k}_{\mathrm{j}}, \mathrm{j}=1, \ldots, \mathrm{J}$. Com a obtenção dos pesos $\mathrm{k}_{\mathrm{j}}$, dispõe-se de todos os elementos para o cálculo de $\omega$, para escalas com qualquer número de categorias. Na Tabela 2 são apresentados os valores numéricos dos pesos $\mathrm{k}_{\mathrm{j}}$ para escalas de $\mathrm{J}+1=3$ a até $\mathrm{J}+1=12$ categorias por serem estes tamanhos de escala observados em experimentos de avaliação de doenças em plantas. Na prática, o que é calculado é $\omega$ e, em experimentos, as análises são efetuadas com esta medida que corresponde à transformação angular do índice de infecção I de fato associado à unidade experimental. Os resultados e conclusões referentes às médias de tratamentos são então expressas na escala original de I, pela transformação inversa $\overline{\mathrm{I}}_{\mathrm{t}}=\operatorname{sen}^{2} \overline{\omega_{\mathrm{t}}}$.

\section{APLICAÇÃ̃O}

Para ilustrar o cálculo e aplicação do índice de intensidade de infecção, foram utilizados os resultados de dois experimentos desenvolvidos na 
Embrapa-Centro Nacional de Pesquisa de Uva e Vinho (CNPUV), ambos delineados em blocos completos com parcelas casualizadas.

No ensaio 1, foram pesquisados os efeitos de doze métodos de poda verde $(\mathrm{T} 1, \mathrm{~T} 2, \ldots, \mathrm{T} 12)$ sobre a sanidade da uva 'Merlot'. Procedeu-se à avaliação visual de 50 cachos por parcela, nas três repetições por tratamento, seguindo-se escala de seis classes de severidade a saber: $\mathrm{x}_{0}=0$ ou "sem sintoma"; $\mathrm{x}_{1}=1$ ou " 1 a $5 \%$ de podridão"; $\mathrm{x}_{2}=2$ ou " 5 a $10 \%$ de podridão"; $\mathrm{x}_{3}=3$ ou " 10 a $25 \%$ de podridão"; $\mathrm{x}_{4}=4$ ou " 25 a $50 \%$ de podridão" e $\mathrm{x}_{5}=5$ ou "mais de $50 \%$ de podridão". Os resultados da contagem de cachos estão na Tabela 3, com o resultado do cálculo de $\omega$ e da média do índice I por tratamento. No ensaio 2, foram aplicados dez métodos de desfolha (D1, D2,..,D10), em quatro repetições de plantas da cultivar Pinot Noir e foram avaliados, quanto à severidade de doença, todos os cachos da parcela, seguindo-se a mesma escala do ensaio 1, resultando nas distribuições de freqüências e valores de $\omega$ e da média de I da Tabela 4 . Nos dois ensaios, foram calculados também os índices de doença (ID) segundo a fórmula de McKinney (1923) (Tabelas 3 e 4), a fim de se efetuarem comparações entre $\omega$ e ID.

Utilizando-se os dados das três repetições do tratamento 1 do ensaio 1 , são apresentadas planilhas mostrando as etapas para a obtenção de $\omega$ (Tabelas 5 e 6). A bateria de cálculos pode parecer pesada, mas os computadores a tornam simples e rápida, seja por meio de planilhas de cálculo, ou por meio de aplicativos como o SAS (SAS Institute, 1990), utilizado neste trabalho.

Para verificar a validade de $\omega$ e do ID numa análise de variância, procedeu-se aos testes das hipóteses de normalidade dos erros experimentais e de homogeneidade de variâncias de tratamentos. Foram obtidas as estimativas dos erros experimentais (resíduos) associados a cada medida, com base no modelo do delineamento adotado. Nos testes de normalidade, usaram-se diagramas de dispersão dos resíduos padronizados contra quantis normais, e o teste do coeficiente de correlação, entre essas medidas (Filliben, 1975).

TABELA 2. Pesos $k_{\mathrm{j}}$, utilizados no cálculo de $\omega$, de acordo com o número de classes referentes à presença de infecção, para escalas de três a doze categorias.

\begin{tabular}{rcccccccccc}
\hline $\mathrm{J}=1, \ldots, \mathrm{J}$ & \multicolumn{8}{c}{ Número de classes referentes à presença de infecção $(\mathrm{J})$} \\
\cline { 2 - 10 } & 2 & 3 & 4 & 5 & 6 & 7 & 8 & 9 & 10 & 11 \\
\hline 1 & 0,5 & 0,392 & 0,333 & 0,295 & 0,268 & 0,247 & 0,230 & 0,216 & 0,205 & 0,195 \\
2 & 0,5 & 0,216 & 0,167 & 0,141 & 0,124 & 0,112 & 0,103 & 0,096 & 0,090 & 0,085 \\
3 & & 0,392 & 0,167 & 0,128 & 0,108 & 0,095 & 0,086 & 0,079 & 0,074 & 0,069 \\
4 & & & 0,333 & 0,141 & 0,108 & 0,091 & 0,080 & 0,073 & 0,067 & 0,062 \\
5 & & & & 0,295 & 0,124 & 0,095 & 0,080 & 0,071 & 0,064 & 0,059 \\
6 & & & & & 0,268 & 0,112 & 0,086 & 0,073 & 0,064 & 0,058 \\
7 & & & & & & 0,247 & 0,103 & 0,079 & 0,067 & 0,059 \\
8 & & & & & & & 0,230 & 0,096 & 0,074 & 0,062 \\
9 & & & & & & & & 0,216 & 0,090 & 0,069 \\
10 & & & & & & & & & 0,205 & 0,085 \\
11 & & & & & & & & & & 0,195 \\
\hline
\end{tabular}


Para verificar a homogeneidade de variâncias, usou-se o teste de Bartlett (Silva, 1969).

Nas Figs. 1 e 2 estão os gráficos de resíduos padronizados (A - associados ao $\omega$ e $\mathrm{B}$ - associados ao ID) contra os quantis normais correspondentes, nos ensaios 1 e 2 , respectivamente. Pelo julgamento visual dos gráficos A, supôs-se a normalidade dos erros associados às observações $\omega$, em am-

TABELA 3. Número de cachos por classe de severidade de doença, valores de $\omega$ por parcela, médias do índice de infecção por tratamento $\left(\overline{I_{t}}\right)$ e índice de doença (ID) por parcela, para o ensaio 1.

\begin{tabular}{|c|c|c|c|c|c|c|c|c|c|c|}
\hline \multirow[t]{2}{*}{ Tratamento } & \multirow[t]{2}{*}{ Bloco } & \multicolumn{6}{|c|}{$\begin{array}{l}\text { Número de cachos }\left(\mathrm{F}_{\mathrm{j}}\right) \\
\text { por classe de severidade }\left(\mathrm{x}_{\mathrm{j}}\right)\end{array}$} & \multirow[t]{2}{*}{$\omega$} & \multirow[t]{2}{*}{$\overline{I_{t}}=\operatorname{sen}^{2} \overline{\omega_{t}}$} & \multirow[t]{2}{*}{ ID } \\
\hline & & $\mathrm{X}_{0}$ & $\mathrm{X}_{1}$ & $\mathrm{x}_{2}$ & $\mathrm{X}_{3}$ & $\mathrm{X}_{4}$ & $\mathrm{X}_{5}$ & & & \\
\hline \multirow[t]{3}{*}{$\mathrm{T} 1$} & 1 & 19 & 9 & 4 & 5 & 6 & 7 & 39,2180 & \multirow[t]{3}{*}{18,78} & 36,4 \\
\hline & 2 & 41 & 4 & 3 & 1 & 0 & 1 & 16,9012 & & 7,2 \\
\hline & 3 & 40 & 6 & 0 & 0 & 2 & 2 & 20,9130 & & 9,6 \\
\hline \multirow[t]{3}{*}{$\mathrm{T} 2$} & 1 & 34 & 4 & 5 & 1 & 2 & 4 & 27,2391 & \multirow[t]{3}{*}{18,93} & 18,0 \\
\hline & 2 & 33 & 8 & 1 & 4 & 3 & 1 & 23,2331 & & 15,6 \\
\hline & 3 & 31 & 7 & 8 & 1 & 0 & 3 & 26,9091 & & 16,4 \\
\hline \multirow[t]{3}{*}{ T3 } & 1 & 36 & 6 & 4 & 2 & 1 & 1 & 19,8423 & \multirow[t]{3}{*}{14,24} & 11,6 \\
\hline & 2 & 37 & 3 & 4 & 4 & 1 & 1 & 20,7335 & & 12,8 \\
\hline & 3 & 31 & 10 & 3 & 1 & 2 & 3 & 25,9350 & & 16,8 \\
\hline \multirow[t]{3}{*}{$\mathrm{T} 4$} & 1 & 36 & 11 & 2 & 0 & 1 & 0 & 14,3822 & \multirow[t]{3}{*}{6,23} & 7,6 \\
\hline & 2 & 45 & 4 & 1 & 0 & 0 & 0 & 6,6237 & & 2,4 \\
\hline & 3 & 36 & 6 & 3 & 1 & 2 & 2 & 22,3457 & & 13,2 \\
\hline \multirow[t]{3}{*}{ T5 } & 1 & 34 & 10 & 1 & 2 & 2 & 1 & 20,9105 & \multirow[t]{3}{*}{17,50} & 9,6 \\
\hline & 2 & 31 & 7 & 6 & 2 & 1 & 3 & 26,3027 & & 11,6 \\
\hline & 3 & 28 & 11 & 3 & 2 & 4 & 2 & 26,9683 & & 10,8 \\
\hline \multirow[t]{3}{*}{ T6 } & 1 & 20 & 16 & 5 & 4 & 2 & 3 & 30,9141 & \multirow[t]{3}{*}{21,77} & 24,4 \\
\hline & 2 & 35 & 6 & 4 & 3 & 0 & 2 & 23,3962 & & 13,2 \\
\hline & 3 & 32 & 7 & 2 & 3 & 1 & 5 & 29,1256 & & 19,6 \\
\hline \multirow[t]{3}{*}{$\mathrm{T} 7$} & 1 & 33 & 4 & 4 & 5 & 3 & 1 & 24,2144 & \multirow[t]{3}{*}{20,57} & 17,6 \\
\hline & 2 & 26 & 9 & 4 & 6 & 1 & 4 & 30,8933 & & 23,6 \\
\hline & 3 & 38 & 2 & 2 & 2 & 2 & 4 & 25,8134 & & 16,0 \\
\hline \multirow[t]{3}{*}{$\mathrm{T} 8$} & 1 & 29 & 9 & 6 & 1 & 1 & 4 & 28,3628 & \multirow[t]{3}{*}{26,40} & 19,2 \\
\hline & 2 & 27 & 5 & 8 & 2 & 3 & 5 & 32,4101 & & 25,6 \\
\hline & 3 & 32 & 5 & 3 & 2 & 1 & 7 & 31,9770 & & 22,4 \\
\hline \multirow[t]{3}{*}{ T9 } & 1 & 36 & 6 & 5 & 1 & 0 & 2 & 22,2233 & \multirow[t]{3}{*}{9,37} & 11,6 \\
\hline & 2 & 37 & 5 & 4 & 3 & 0 & 1 & 20,0156 & & 10,8 \\
\hline & 3 & 41 & 6 & 1 & 2 & 0 & 0 & 11,2342 & & 5,6 \\
\hline \multirow[t]{3}{*}{$\mathrm{T} 10$} & 1 & 38 & 9 & 1 & 1 & 0 & 1 & 17,4690 & 7,49 & 7,6 \\
\hline & 2 & 43 & 4 & 0 & 1 & 0 & 2 & 18,6650 & & 6,8 \\
\hline & 3 & 44 & 2 & 3 & 0 & 1 & 0 & 11,5300 & & 4,8 \\
\hline T11 & 1 & 32 & 9 & 2 & 1 & 2 & 4 & 27,2144 & 12,51 & 17,6 \\
\hline & 2 & 37 & 8 & 2 & 2 & 0 & 1 & 18,8302 & & 9,2 \\
\hline & 3 & 32 & 11 & 4 & 3 & 0 & 0 & 16,1020 & & 11,2 \\
\hline T12 & 1 & 33 & 3 & 5 & 1 & 1 & 7 & 31,8837 & 28,09 & 22,0 \\
\hline & 2 & 24 & 5 & 7 & 7 & 3 & 4 & 33,4369 & & 28,8 \\
\hline & 3 & 35 & 3 & 3 & 0 & 3 & 6 & 30,6891 & & 20,4 \\
\hline
\end{tabular}

Pesq. agropec. bras., Brasília, v.34, n.9, p.1545-1555, set. 1999 
bos os ensaios. A normalidade foi confirmada pelos coeficientes de correlação: $r_{1}=0,984$ e $r_{2}=0,986$, não-significativos, respectivamente, nos ensaios 1 e 2 (os valores críticos correspondentes são $\rho_{1}=0,969$ e $\rho_{2}=0,972$ para $\alpha=0,05)$. No teste da hipótese de homogeneidade de variâncias de tra-

TABELA 4. Número de cachos por classe de severidade de doença, valores de $\omega$ por parcela, médias do índice de infecção por tratamento $\left(\bar{I}_{t}\right)$ e índice de doença (ID) por parcela, para o ensaio 2.

\begin{tabular}{|c|c|c|c|c|c|c|c|c|c|c|}
\hline \multirow[t]{2}{*}{ Tratamento } & \multirow[t]{2}{*}{ Bloco } & \multicolumn{6}{|c|}{$\begin{array}{l}\text { Número de cachos }\left(\mathrm{F}_{\mathrm{j}}\right) \\
\text { por classe de severidade }\left(\mathrm{x}_{\mathrm{j}}\right)\end{array}$} & \multirow[t]{2}{*}{$\omega$} & \multirow[t]{2}{*}{$\overline{I_{t}}=\operatorname{sen}^{2} \overline{\omega_{t}}$} & \multirow[t]{2}{*}{ ID } \\
\hline & & $\mathrm{x}_{0}$ & $\mathrm{x}_{1}$ & $\mathrm{X}_{2}$ & $\mathrm{X}_{3}$ & $\mathrm{X} 4$ & $\mathrm{X}_{5}$ & & & \\
\hline \multirow[t]{4}{*}{ D1 } & 1 & 17 & 19 & 9 & 11 & 11 & 23 & 49,0703 & \multirow[t]{4}{*}{60,21} & 50,89 \\
\hline & 2 & 12 & 29 & 7 & 8 & 7 & 31 & 52,5828 & & 53,19 \\
\hline & 3 & 7 & 13 & 13 & 13 & 13 & 10 & 48,2519 & & 52,17 \\
\hline & 4 & 2 & 22 & 3 & 7 & 6 & 15 & 53,6712 & & 53,82 \\
\hline \multirow[t]{4}{*}{ D2 } & 1 & 7 & 11 & 8 & 5 & 6 & 9 & 47,1882 & \multirow[t]{4}{*}{57,07} & 48,26 \\
\hline & 2 & 8 & 27 & 12 & 4 & 6 & 16 & 47,7433 & & 45,75 \\
\hline & 3 & 12 & 11 & 9 & 8 & 16 & 9 & 46,2985 & & 49,85 \\
\hline & 4 & 7 & 8 & 4 & 9 & 9 & 18 & 55,0352 & & 61,45 \\
\hline \multirow[t]{4}{*}{ D3 } & 1 & 1 & 6 & 4 & 12 & 6 & 38 & 68,0901 & \multirow[t]{4}{*}{62,35} & 78,81 \\
\hline & 2 & 17 & 11 & 15 & 15 & 5 & 12 & 44,0926 & & 44,27 \\
\hline & 3 & 12 & 15 & 18 & 9 & 18 & 16 & 48,7083 & & 52,27 \\
\hline & 4 & 10 & 19 & 8 & 7 & 3 & 15 & 47,7074 & & 46,13 \\
\hline \multirow[t]{4}{*}{ D4 } & 1 & 2 & 14 & 14 & 9 & 12 & 7 & 49,4113 & \multirow[t]{4}{*}{48,91} & 52,41 \\
\hline & 2 & 6 & 15 & 14 & 15 & 15 & 9 & 48,0801 & & 52,16 \\
\hline & 3 & 12 & 32 & 15 & 8 & 5 & 3 & 35,6990 & & 32,27 \\
\hline & 4 & 9 & 20 & 16 & 9 & 5 & 10 & 44,3217 & & 43,19 \\
\hline \multirow[t]{4}{*}{ D5 } & 1 & 6 & 21 & 7 & 4 & 5 & 12 & 47,8369 & \multirow[t]{4}{*}{64,93} & 46,18 \\
\hline & 2 & 6 & 19 & 11 & 9 & 11 & 36 & 58,0292 & & 63,48 \\
\hline & 3 & 6 & 18 & 18 & 17 & 15 & 53 & 60,3349 & & 67,72 \\
\hline & 4 & 9 & 13 & 7 & 6 & 6 & 13 & 48,5479 & & 49,63 \\
\hline \multirow[t]{4}{*}{ D6 } & 1 & 14 & 19 & 10 & 12 & 9 & 16 & 46,8234 & \multirow[t]{4}{*}{55,42} & 47,75 \\
\hline & 2 & 5 & 13 & 12 & 9 & 8 & 4 & 43,8560 & & 45,49 \\
\hline & 3 & 2 & 21 & 15 & 14 & 15 & 34 & 58,5777 & & 63,96 \\
\hline & 4 & 9 & 16 & 15 & 9 & 3 & 8 & 43,1886 & & 41,67 \\
\hline \multirow[t]{4}{*}{ D7 } & 1 & 18 & 19 & 11 & 4 & 3 & 14 & 43,0848 & \multirow[t]{4}{*}{35,30} & 39,13 \\
\hline & 2 & 22 & 30 & 11 & 9 & 15 & 6 & 38,1327 & & 36,34 \\
\hline & 3 & 10 & 8 & 8 & 8 & 2 & 0 & 30,2504 & & 31,11 \\
\hline & 4 & 25 & 15 & 3 & 1 & 3 & 7 & 34,3377 & & 26,30 \\
\hline \multirow[t]{4}{*}{ D8 } & 1 & 1 & 0 & 2 & 9 & 10 & 52 & 74,8731 & \multirow[t]{4}{*}{80,56} & 89,46 \\
\hline & 2 & 4 & 9 & 18 & 18 & 15 & 27 & 57,2483 & & 64,62 \\
\hline & 3 & 7 & 9 & 7 & 9 & 8 & 38 & 61,3753 & & 69,74 \\
\hline & 4 & 2 & 10 & 8 & 5 & 16 & 28 & 61,8614 & & 71,01 \\
\hline \multirow[t]{4}{*}{ D9 } & 1 & 1 & 3 & 9 & 10 & 14 & 44 & 68,2868 & 65,65 & 80,74 \\
\hline & 2 & 23 & 33 & 20 & 15 & 10 & 25 & 45,6845 & & 44,92 \\
\hline & 3 & 7 & 10 & 8 & 8 & 4 & 28 & 58,2270 & & 63,38 \\
\hline & 4 & 22 & 17 & 12 & 9 & 7 & 17 & 44,2745 & & 43,10 \\
\hline D10 & 1 & 3 & 14 & 8 & 14 & 16 & 30 & 59,3753 & 64,22 & 67,29 \\
\hline & 2 & 2 & 7 & 6 & 9 & 7 & 22 & 61,1416 & & 69,43 \\
\hline & 3 & 30 & 27 & 21 & 16 & 13 & 23 & 44,1193 & & 43,69 \\
\hline & 4 & 9 & 19 & 17 & 11 & 17 & 14 & 48,3841 & & 51,49 \\
\hline
\end{tabular}


tamentos, com a variável $\omega$, obteve-se $\chi^{2}$ igual a 13,06 e 7,62, respectivamente nos ensaios 1 e 2 . Os respectivos valores críticos são $\chi^{2}=19,68$ ( 11 graus de liberdade e $\alpha=0,05$ ) e $\chi^{2}=16,92$ ( 9 graus de liberdade e $\alpha=0,05$ ). Portanto, $\omega$ cumpre as pressuposições para se efetuar análise da variação em ambos os ensaios.

A observação das Figs. 1B e 2B levou a suspeitar da falta de normalidade dos resíduos associados ao ID, no ensaio 1 . De fato, nesse ensaio, a hipótese distribucional de normalidade foi rejeitada $(\mathrm{r}=0,963$ significativo para $\alpha=0,05)$. A hipótese de homogeneidade de variâncias de tratamentos não foi rejeitada em nenhum dos ensaios, se considerado o nível de significância de 0,05 (os valores calculados de $\chi^{2}$ foram 19,00 no ensaio 1 e 14,22 no ensaio 2). No ensaio 1, se ignorada a transgressão ao modelo adotado e efetuada a análise de variação, essa e seus desdobramentos levarão a inferências não verdadeiras no âmbito do ensaio.

Assim como a média ponderada, o ID, usado por pesquisadores em fitossanidade, depende dos valores da escala adotada, os quais são arbitrários, isto é, se estabelecida uma escala de notas igual a $0,1,2$ e 3 ou a 1, 2, 3 e 4 (não iniciada em zero) ou a $0,1,3$ e 5 (valores não equidistantes), todas para representar, respectivamente, "ausência de infecção", "infecção fraca", "infecção regular" e "infecção forte", uma classificação idêntica de

TABELA 5. Planilha com etapas iniciais do cálculo de $\omega$ (freqüências absolutas e relativas e estimativas de probabilidades) para as repetições do tratamento $\mathrm{T} 1$ do ensaio 1.

\begin{tabular}{|c|c|c|c|c|c|c|c|c|c|c|c|c|c|c|c|}
\hline \multirow[t]{2}{*}{ Bloco } & \multicolumn{6}{|c|}{ Freqüências absolutas $\left(\mathrm{F}_{\mathrm{j}}\right)$} & \multirow[t]{2}{*}{$\mathrm{n}$} & \multicolumn{8}{|c|}{$\begin{array}{c}\text { Freqüências relativas e estimativas de } \\
\text { probabilidades }\end{array}$} \\
\hline & $\mathrm{F}_{0}$ & $\mathrm{~F}_{1}$ & $\mathrm{~F}_{2}$ & $\mathrm{~F}_{3}$ & $\mathrm{~F}_{4}$ & $\mathrm{~F}_{5}$ & & $\mathrm{f}_{1}=\mathrm{p}_{1,5}$ & $\mathrm{f}_{2}$ & $f_{3}$ & $\mathrm{f}_{4}$ & $\mathrm{f}_{5}$ & $\mathrm{p}_{2,5}$ & $\mathrm{p}_{3,5}$ & $\mathrm{p}_{4,5}$ \\
\hline 1 & 19 & 9 & 4 & 5 & 6 & 7 & 50 & 0,62 & 0,71 & 0,82 & 0,72 & 0,54 & 0,44 & 0,36 & 0,26 \\
\hline 2 & 41 & 4 & 3 & 1 & 0 & 1 & 50 & 0,18 & 0,56 & 0,40 & 0,50 & 1,00 & 0,10 & 0,04 & 0,02 \\
\hline 3 & 40 & 6 & 0 & 0 & 2 & 2 & 50 & 0,20 & 0,40 & 1,00 & 1,00 & 0,50 & 0,08 & 0,08 & 0,08 \\
\hline
\end{tabular}

TABELA 6. Planilha com etapas finais do cálculo de $\omega\left(\operatorname{arcsen} \sqrt{f_{j}}\right.$, $\mathbf{k}_{\mathrm{j}} \sqrt{\mathbf{p}_{\mathrm{j}-1,5}} \operatorname{arcsen} \sqrt{\mathbf{f}_{\mathrm{j}}}$ e $\omega$ ) para as repetições do tratamento $\mathrm{T} 1$ do ensaio 1.

\begin{tabular}{|c|c|c|c|c|c|c|c|c|c|c|c|}
\hline \multirow[t]{2}{*}{ Bloco } & \multicolumn{5}{|c|}{$\operatorname{arcsen} \sqrt{f_{j}} 1$} & \multicolumn{5}{|c|}{$\mathrm{k}_{\mathrm{j}} \sqrt{\mathrm{p}_{\mathrm{j}-1,5}} \operatorname{arcsen}{\sqrt{\mathrm{f}_{\mathrm{j}}}}^{2}$} & \multirow[t]{2}{*}{$\omega^{3}$} \\
\hline & $j=1$ & $j=2$ & $j=3$ & $j=4$ & $\mathrm{j}=5$ & $\mathrm{j}=1$ & $\mathrm{j}=2$ & $j=3$ & $\mathrm{j}=4$ & $j=5$ & \\
\hline 1 & 51,94 & 57,40 & 64,76 & 58,19 & 47,21 & 15,32 & 6,37 & 5,50 & 4,92 & 7,10 & 39,22 \\
\hline 2 & 25,10 & 48,19 & 39,23 & 45,00 & 90,00 & 7,41 & 2,88 & 1,59 & 1,27 & 3,75 & 16,90 \\
\hline 3 & 26,57 & 39,23 & 90,00 & 90,00 & 45,00 & 7,84 & 2,47 & 3,26 & 3,59 & 3,75 & 20,91 \\
\hline
\end{tabular}

1 Arco seno da raiz quadrada da freqüência relativa $\left(f_{j}\right)$ obtida na Tabela 5 , com o $j$ correspondente.

${ }^{2}$ Pesos $\mathrm{k}_{\mathrm{j}}$ obtidos na Tabela 2 para $\mathrm{J}=5$ e freqüências relativas $\left(\mathrm{f}_{\mathrm{j}}\right)$ e estimativas de probabilidades $\left(\mathrm{p}_{\mathrm{j}-1,5}\right)$ obtidas na Tabela 5 , com o j correspondente.

$3 \omega=\sum_{\mathrm{j}=1}^{5} \mathrm{k}_{\mathrm{j}} \sqrt{\mathrm{p}_{\mathrm{j}-1,5}} \operatorname{arcsen} \sqrt{\mathrm{f}_{\mathrm{j}}}$. 
n indivíduos levará a diferentes valores percentuais da medida de infecção. Por outro lado, obedecida a mesma escala de avaliação, diferentes distribuições de $\mathrm{n}$ indivíduos resultam no mesmo ID. As realizações dessa variável são múltiplos de $1 /\left(\mathrm{n} \mathrm{x}_{\mathrm{J}}\right)$, no intervalo [0;100], o que não a caracteriza como variável contínua. As duas últimas afirmativas podem ser mais facilmente observadas com os dados do ensaio 1 (Tabela 3), onde o número $n$ de indivíduos (cachos, nesse caso) é o mesmo em todas as parcelas.

Com a determinação do método de obtenção dos pesos $\mathrm{k}_{\mathrm{j}}$, necessários para o cálculo de $\omega$, pode se obter essa medida para qualquer tamanho $\mathrm{J}+1$ de escala. E como esse cálculo não envolve os valores ou notas da escala adotada, $\omega$ pode ser aplicado a escalas qualitativas ordinais. Essa transformação angular do índice de intensidade de infecção I cumpre os requisitos de normalidade e de homogeneidade de variâncias de tratamentos, e pode ser submetida à análise de variação e procedimentos de inferência subseqüentes.
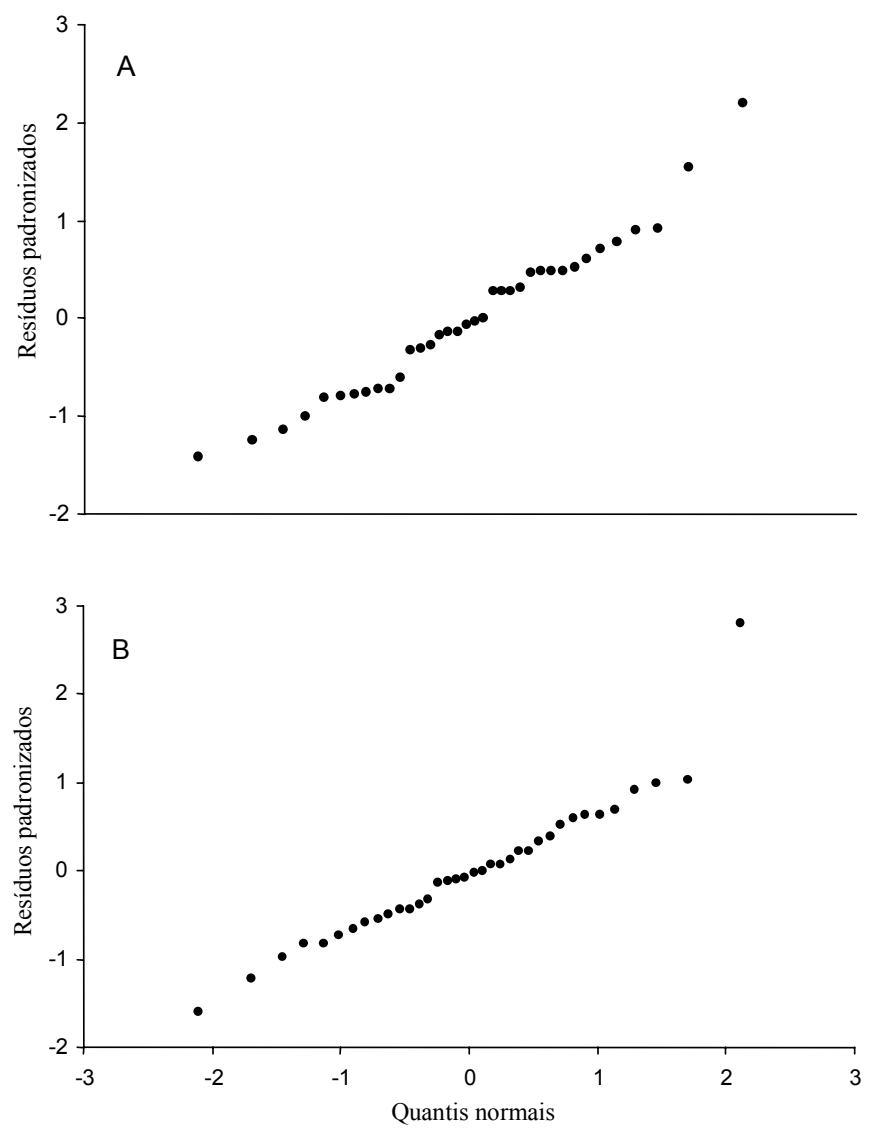

FIG. 1. Diagrama de dispersão dos resíduos padronizados associados a $\omega$ (A) e ao ID (B) contra quantis normais, no ensaio 1. 

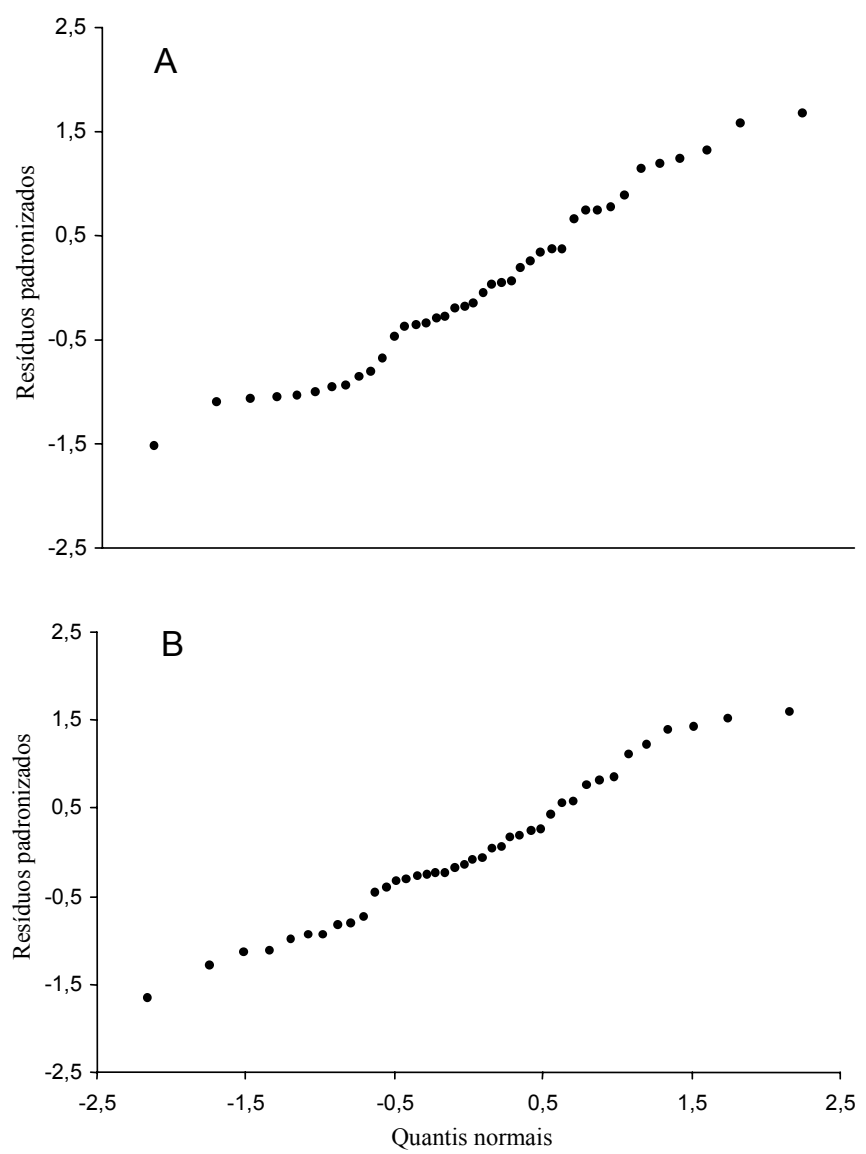

FIG. 2. Diagrama de dispersão dos resíduos padronizados associados a $\omega$ (A) e ao ID (B) contra quantis normais, no ensaio 2.

\section{AGRADECIMENTOS}

Ao pesquisador Francisco Mandelli, da Embrapa-CNPUV, por ceder dados de experimentos sob sua responsabilidade.

\section{REFERÊNCIAS}

AMARAL, E. Novo índice de intensidade de infecção. Pesquisa Agropecuária Brasileira: Série Agronomia, Rio de Janeiro, v.4, n.2, p.1-2, 1969.

BARTLETT, M.S. The use of transformations. Biometrics, v.3, p.39-52, 1947.

COCHRAN, W.G. Analysis of variance for percentages based on unequal numbers. Journal of the American Statistical Association, v.38, p.287-301, 1943. 
ESKRIDGE, K.M. Statistical analysis of disease reaction data using nonparametric methods. HortScience, v.30, p.478-481, June 1995.

FILLIBEN, J.J. The probability plot correlation coefficient test for normality. Technometrics, Washington, v.17, p.111-117, 1975.

HUGHES, G.; MADDEN, L.V. Some methods allowing for aggregated patterns of disease incidence in the analysis of data from designed experiments. Plant Pathology, v.44, p.927-943, 1995.

McKINNEY, H.H. Influence of soil, temperature and moisture on infection of wheat seedlings by Helminthosporium sativum. Journal of Agricultural Research, Washington, v.26, p.195-217, Nov. 1923.

SAS INSTITUTE. SAS language: reference. version 6. Cary, 1990. 1042p.

SEEM, R.C. Disease incidence and severity relationships. Annual Review of Phytopathology, Palo Alto, v.22, p.133-150, 1984.

SILVA, J.G.C. da. Análise estatística de um novo índice de intensidade de infecção. Pesquisa Agropecuária Brasileira: Série Agronomia, Rio de Janeiro, v.4, n.2, p.3-7, 1969. 